\title{
De Raisel à Tourvel. Mme Riccoboni et les futures Liaisons dangereuses de Laclos
}

\section{Flavien Falantin}

\section{(2) OpenEdition}

Journals

Édition électronique

URL : http://journals.openedition.org/rief/1030

DOI : 10.4000/rief.1030

ISSN : 2240-7456

Éditeur

Seminario di filologia francese

Référence électronique

Flavien Falantin, «De Raisel à Tourvel. Mme Riccoboni et les futures Liaisons dangereuses de Laclos », Revue italienne d'études françaises [En ligne], 5 | 2015, mis en ligne le 15 décembre 2015, consulté le 21 avril 2019. URL : http://journals.openedition.org/rief/1030 ; DOl : 10.4000/rief.1030

Ce document a été généré automatiquement le 21 avril 2019.

\section{(c) (i) (9)}

Les contenus de la RIEF sont mis à disposition selon les termes de la Licence Creative Commons Attribution - Pas d'Utilisation Commerciale - Pas de Modification 4.0 International. 


\title{
De Raisel à Tourvel. Mme Riccoboni et les futures Liaisons dangereuses de Laclos
}

\author{
Flavien Falantin
}

1 La correspondance entre Laclos et Mme Riccoboni, commencée par cette dernière en 1782 suite à la parution des Liaisons dangereuses, a fait couler beaucoup d'encre'. ${ }^{1}$. Mais qu'a-t-on réellement retenu de leur échange? Les études sur la joute à laquelle se sont livrés les épistoliers ont surtout fait mention des offuscations pudibondes de la romancière à l'égard de l'invraisemblable Merteuil ${ }^{2}$, mais aussi de la place et du rôle tant moral qu'éducatif de l'écrivain. Cependant plusieurs éléments de l'analyse semblent avoir été oubliés. Tout d'abord le ton inquisiteur - et peu habituel de Mme Riccoboni ${ }^{3}$ - évoque davantage celui d'un procureur que celui d'une philosophe stricto sensu. Puis, vient l'attitude de Laclos qui, aux prises avec ce «tribunal »", se justifie parfois d'une manière embarrassée allant jusqu'à confondre sa défense personnelle avec celle de son livre. Mais c'est surtout en présence de deux phrases anodines, lancées à la dérobée par la "plaignante », que l'idée du procès fait son apparition, "vous me feriez un tort véritable en m'attribuant la partialité d'un auteur. Je le suis de si peu de choses qu'en lisant un livre nouveau je me trouverais bien injuste et bien sotte, si je le comparais aux bagatelles sorties de ma plume et croyais mes idées propres à guider celles des autres $\|^{5}$. Il se cache à n'en pas douter derrière la feinte modestie de ces phrases des insinuations revendicatrices plus ambiguës. Bien que la postérité ait injustement plongé Mme Riccoboni dans l'oubli comme le soulignait Emily Crosby ${ }^{6}$ - il n'en demeure pas moins que « les bagatelles sorties de sa plume " représentent à l'époque de véritables succès de vente en France et en Angleterre. Quant à ses contributions littéraires, l'histoire prouve qu'elles ont " guid[é] celles des autres » : son travail de réécriture, lorsqu'elle rédige la fin de La Vie de Marianne, restée inachevée, fut si talentueux que des précisions ont été nécessaires pour pleinement le distinguer de l'œuvre de Marivaux ${ }^{7}$. Figure incontournable du milieu littéraire, la romancière participe de manière active au débat de son temps en fréquentant les plus grands penseurs de l'époque, et tout particulièrement David Hume. Enfin n'oublions pas 
que l'admiration de Laclos pour son roman L'Histoire d'Ernestine, lui inspire une adaptation au théâtre en 1777.

En définitive, les contre-vérités énoncées dans ces deux phrases traduisent une double ironie. La modestie qui entoure cette dépréciation féminine sous-entend en filigrane une raillerie soulignant le manque de considération envers les femmes dont elle est ellemême victime. Corollairement, le second argument repose sur l'idée qu'à la fin de sa vie et forte de sa notoriété, la romancière a probablement voulu régler un contentieux plus large avec Laclos, dépassant le simple cas de Merteuil. Trahirait-elle ici l'idée selon laquelle ses propres livres auraient pu guider Laclos? Opérait-elle une comparaison pour reprendre ses termes - entre l'un de ses livres et les Liaisons dangereuses? Partant du postulat que ce sarcasme renfermerait l'objet de la véritable querelle, alors le différend de la romancière avec Laclos se situerait hors de la lettre de cet échange. Selon nous, la querelle se loge au cœur de l'analyse des personnages de sa fiction toute entière; des créatures d'ailleurs fort ressemblantes à celles décrites vingt-quatre ans plus tôt dans l' Histoire de M. le marquis de Cressy, par une dénommée Marie-Jeanne de Heurles Laboras de Mézières ${ }^{8}$.

Dans cette nouvelle parue en 1758, Riccoboni raconte l'histoire d'un audacieux marquis en quête d'une femme pouvant lui assurer la fortune et la position sociale à laquelle il aspire. Très vite, il se retrouve tiraillé entre deux cœurs amis, celui de la jeune Adélaïde du Bugei, ignorante des passions, et celui de la comtesse de Raisel, veuve et de condition supérieure. Après avoir fait une cour assidue à Mlle du Bugei, qu'il trahit en poussant trop loin l'exercice de la galanterie, le marquis finit par épouser Mme de Raisel. L'escalade des machinations de Cressy pousse Adélaïde à rentrer dans les ordres et sa propre femme à se suicider. Non sans un certain mimétisme Laclos réunit la correspondance d'un couple de libertins sans scrupule, la marquise de Merteuil et le vicomte de Valmont, qui se jouent de l'innocence de la jeune Cécile de Volanges et de la pieuse Présidente de Tourvel. Le roman s'achève sur la prise du voile de la première et sur la folie mortelle de la seconde.

\section{Une autre vision du roman}

Malgré ces ressemblances déroutantes dans l'intrigue, la logique littéraire de Laclos ne s'inscrit pas dans la droite ligne présentée par Riccoboni à son lecteur. La conception de son ouvrage est aux antipodes de celle voulue par l'auteure de l'Histoire de M. le marquis de Cressy. Riccoboni connaît un succès retentissant en publiant un roman qui s'inspire directement des livres présentés au public d'une époque où, selon Georges May, «tout le monde s'entendait à faire du roman un instrument de prédication morale ${ }^{9}$. Comme le précise Martine Reid dans sa présentation, l'Histoire de M. le marquis de Cressy ressemble davantage à un apologue éducatif plein de manichéisme, "ses attaques prennent volontiers la forme de maximes [...]. Pour quelques liaisons heureuses, pour quelques mariages bien assortis, pour quelques hommes vraiment sensibles, combien d'unions mal assorties, d'hommes retors et de femmes trompées $! »^{10}$.

5 Dans la continuation des œuvres de Richardson, le récit de Riccoboni est profondément axé sur une division stricte des champs d'action entre les sexes. Pour reprendre Pierre Fauchery, le romanesque européen incarne par excellence pendant deux siècles «l'odyssée de la conscience sexuée ${ }^{11}$ et la société du roman moral est une société qui, probablement plus que les autres, opère des distinctions fondamentales quant aux sexes 
et à leurs rôles. Le roman libertin cependant offre la vision d'une société profondément sexuelle, où les discours rejoignent les mœurs pour voir dans l'autre sexe un égal. En témoigne la reprise de l'adage de Rousseau en épigraphe des Liaisons dangereuses, «j'ai vu les mœurs de mon temps, et j'ai publié ces lettres ». Le roman épistolaire ne représente pas un instrument narratif neutre, puisqu'il coïncide avec la logique des écrits intimes et spontanés, moins gênés par l'immixtion d'un narrateur engagé.

6 À contrario, le récit de l'Histoire de M. le marquis de Cressy s'enchaîne sous le point de vue d'un observateur omniscient qui, à quelques reprises, intervient de façon non voilée. L'un des angles d'attaque est caractérisé par le jeu d'une écriture féminine qui sert à montrer l'emprise des hommes sur les femmes: «un avantage de la supériorité de l'âme d'un homme [...] dont il se sert pour étouffer les remords qu'élève au fond de son cœur le souvenir d'une femme sensible et malheureuse $»^{12}$. Plus loin encore, le narrateur intervient directement dans le texte pour tenir un discours voisin: "Les hommes s'épargneraient la plus grande partie des peines qu'ils se donnent pour nous en imposer, s'ils pouvaient s'imaginer combien la noblesse de nos idées leur donne de facilité pour nous tromper $»^{13}$.

7 La narratrice joue ici un rôle prépondérant dans la démonstration des préjugés à l'encontre des femmes. De façon assez symptomatique, l'auteure accentue la soumission des femmes vis-à-vis du sexe fort : par deux fois dans le roman, Mme de Raisel et Mlle du Bugei vont baisser les yeux. En effet, quand Adélaïde danse avec le marquis de Cressy « elle baiss[e] les yeux, et n'osa plus les lever $»^{14}$. À son tour, Mme de Raisel «baiss[e] les yeux $»^{15}$ lorsque le marquis lui raconte sa vérité sur le départ d'Adélaïde au couvent. Il est aussi intéressant de noter que dans sa correspondance avec Laclos, Mme Riccoboni réaffirme avec fierté que "c'est en qualité de femme [qu'elle écrit], Monsieur, de Française, de patriote zélée pour l'honneur de [s]a nation $»^{16}$. Fort du bien-fondé de son œuvre, Laclos lui répond : "vous êtes femme et Française ! Hé bien ! ces deux qualités ne m'effraient point. Je sens dans mon cœur tout ce qu'il faut pour ne pas redouter ce tribunal $»^{17}$. Laclos ne craint pas les jugements accablant son éventuelle misogynie car son livre ne brime aucun sexe en particulier. Son ambition est plus universelle, il règle ses comptes avec la société aristocratique tout entière. Il dénonce les perversions des grands seigneurs à travers le prisme de son couple infernal de "scélérats méthodiques " ${ }^{18}$. Grâce au genre épistolaire, une distance s'établit entre le narrateur et l'histoire. Les Liaisons dangereuses représentent un libelle sur les mœurs de l'époque, décrivant l'égale monstruosité des sexes ${ }^{19}$.

Dès lors Riccoboni s'offense de la personnalité monstrueuse de Merteuil. Une femme dotée de tels vices lui semble inconcevable: «on n'a pas besoin de se mettre en garde contre les caractères qui ne peuvent exister $»^{20}$. Le débat est donc lancé sur la vraisemblance de la marquise. Sur ce premier point, Laclos ne parait pas surpris, « on insiste, et l'on me demande, Mme de Merteuil a-t-elle jamais existé ? Je l'ignore ${ }^{21}$. Bien que d'autres prétendent le contraire, Laclos reste évasif sur l'existence d'une femme aussi maléfique. Est-elle sortie de son imagination ou fait-elle réellement partie de ses connaissances $^{22}$ ? Devant cet évitement, l'agacement de Riccoboni pousse Laclos dans ses retranchements et il lui écrit: «je m'applaudis d'avoir passé mes jours dans un petit cercle, et je plains ceux qui étendent assez leurs connaissances pour se rencontrer avec de pareils monstres $»^{23}$. Ce à quoi Laclos répond sur la défensive : "Mais, poursuit-on, vous créez des monstres pour les combattre; de telles femmes n'existent point. Supposons-le, j'y consens : alors, pourquoi tant de rumeur ? $\aleph^{24}$. Laclos se montre plus virulent devant 
l'incrédulité de sa destinatrice et prend le parti de démentir l'hypocrisie selon laquelle le roman doit ressembler à un petit traité de savoir-vivre. Il remet donc Riccoboni dans les cordes de cette sensibilité vertueuse, lorsque cette dernière lui explique qu'un écrivain doit respecter deux principes « celui de plaire, et celui d'être utile. En remplir un, ce n'est pas assez pour un homme honnête ${ }^{25}$. Mais à contre-courant de la philosophie d'Horace, Laclos lui rappelle que l'utilité même de son œuvre provient précisément du soin employé à décrire la nature des Hommes, tout en se permettant au passage de critiquer le travail de son interlocutrice :

Quand ses lecteurs, fatigués de ces images attristantes, voudront se reposer sur des sentiments plus doux ; [...] M. de Laclos les invitera à lire Ernestine, Fanny, Catesby, [...] Peut-être alors conviendront-ils que c'est aux femmes seules, qu'appartiennent cette sensibilité précieuse, cette imagination facile et riante qui embellit tout ce qu'elle touche. ${ }^{26}$

9 Visiblement Laclos met un point d'honneur à défendre l'entreprise de son œuvre. S'il s'attaque à la rigidité de la romancière qui s'impose comme l'arbitre des élégances, l'énumération des œuvres de Mme Riccoboni est toutefois significative de deux choses visibles. La première s'attache à démontrer la candeur de la vertu que l'on retrouve dans l'œuvre de la romancière et qui pourrait expliquer son exaspération devant la sulfureuse Merteuil. La seconde prouve qu'indéniablement Laclos a bien lu Riccoboni. Sans surprise, nous rencontrons dans le roman de Riccoboni une Merteuil embellie, dont les vices s'équilibrent et se répartissent à l'aide de deux personnages féminins distincts.

\section{L'assemblage de Merteuil}

Dans son roman, Riccoboni décrit deux portraits de femmes méprisables, ceux de Mme d'Elmont et de Mlle Hortense de Berneil. La physiologie de ces deux femmes élaborée par l'auteure représente le portrait unifié de la future Merteuil. Reprenons tout d'abord les éléments caractéristiques de Mme d'Elmont. Elle est perçue comme une de ces femmes qui miment les actions des hommes et renoncent «à la pudeur, à la modestie, et à la délicatesse de sentiment, qui est la marque distinctive de l'être $»^{27}$. Une philosophie libertine que l'on retrouve point par point chez la marquise de Merteuil, pour qui la conquête et le plaisir ne sont plus l'apanage des hommes. Elle ne se reconnaît pas dans les femmes de son siècle et se situe même en marge de son sexe: "Mais moi, qu'ai-je de commun avec ces femmes inconsidérées ? ${ }^{28}$ Laclos prolonge le mimétisme hédoniste initié par Riccoboni en représentant son héroïne comme l'égale des hommes, voire plus puissante qu'eux, «ces tyrans détrônés [sont] devenus mes esclaves " ${ }^{29}$. Sous ses airs de femme vertueuse et respectable, se cache une intrigante qui multiplie les amants et qui, "pour éviter de leur paraître volage", leur assure l'exclusivité afin d'obtenir «leur discrétion $»^{30}$. Cette citation révèle la conduite "volage » de la marquise et revient aussi sur son implacable talent de dissimulatrice experte. Il s'agit d'une stratégie de comédienne dont est cruellement dépourvue la pauvre Mme d'Elmont dans son rôle de simple dépravée. Afin de ne pas brusquer ses lecteurs, Riccoboni ne pouvait pas la doter en sus du contrôle de ses attitudes et de ses discours. En confiant cet attribut à une autre figure féminine méprisable, elle ne rompt pas avec sa logique et remplit son contrat narratif de vraisemblance. Les manipulations et le caractère retors du personnage d'Hortense de Berneil, décrite comme une femme glaciale et dissimulatrice, jouent l'autre moitié du Janus-Merteuil. Le portrait d'Hortense indique qu' "Il était difficile de la 
connaître ; un air froid et le silence qu'elle gardait sur ses goûts la faisaient paraître d'une extrême indifférence $»^{31}$.

Le dédoublement des héroïnes effectué par Riccoboni se retrouve unifié par Laclos qui revendique dans sa correspondance l'assemblage de ces deux types de créatures : «j'ai répandu l'alarme et dévoilé leurs coupables artifices [...]. Vous ne me soupçonnerez pas, sans doute, de me comparer à Molière : mais j'ai pu comme lui, rassembler dans un même personnage, les traits épars du même caractère $»^{32}$. Cette alliance de débauche et d'observation, ce « retour sur sa propre personne pour mieux discipliner ses relations sociales et mondaines ${ }^{33}$, dont sont privées les deux héroïnes de Riccoboni, les distinguent de Merteuil qui concentre toutes ces inconduites. Bien qu'il n'y ait pas, à proprement parler, d'équivalence directe de la marquise de Merteuil dans l'Histoire de $M$. le marquis de Cressy, il apparaît cependant plausible que Laclos a pu s'inspirer de la duplicité de Mmes d'Elmont et de Berneil (l'association onomastique ne relève sans doute pas du hasard). Quant à l'analyse psychologique de ces deux personnages, elle démontre l'idée d' "exactitude et [de] fidélité " $^{34}$ dont parle Laclos à Riccoboni, si bien que ce partage équitable du vice entre les sexes n'est pas rendu par la romancière qui se cantonne à brimer l'unique figure masculine de son roman.

\section{Le séquençage de Cressy}

12 Le personnage du marquis de Cressy, principale et seule figure masculine de la nouvelle, concentre tous les malheurs imputables à l'histoire. L'intrigue est entièrement fondée sur le caractère monstrueux du jeune homme. Il incarne cette vieille lignée de "grand seigneur méchant homme $\aleph^{35}$ dont parle Molière, qui se plaît à assouvir ses désirs dans une société corsetée dans des codes moraux. Son ambition destructrice apparaît dans «un désir ardent de s'avancer, d'effacer les autres, et de parvenir à la plus haute fortune ${ }^{36}$. Le triomphe du libertin dépeint par Riccoboni est de s'assurer la sympathie d'une société éprise d'honorabilité tout en étant un parfait scélérat. Cette caractéristique décrit parfaitement Valmont qui a aussi «le cœur faux, beaucoup de finesse dans l'esprit, l'art de cacher ses vices et de connaître le faible d'autrui $»^{37}$. Toutefois, la personnalité de Cressy intervient encore comme un agent double puisque sa jeunesse assouplit sa méchanceté, conférant à sa personnalité le feu de la fougue et l'ingénuité de la passion. Cette ambivalence lui donne l'aspect virginal du «beau héros de Roman $»^{38}$ dont parle Valmont au sujet de Danceny. À plusieurs reprises Riccoboni efface la prévalence de son ambition et en supprime la monstruosité, pour montrer les contours d'un cœur plus pur, notamment lorsqu' "il fut tenté de sacrifier tous ses projets de grandeur et de fortune à l'attrait du bonheur véritable qu'il pouvait trouver dans la possession d'une fille charmante dont il était adoré $"^{39}$.

13 Face à l'omnipotence narrative de Cressy, Laclos inverse la courbe homologique de la combinaison opérée sur Merteuil, en séquençant ce héros en deux protagonistes masculins distincts, Valmont et Danceny. Il faut saisir ce désassemblage de Cressy comme un effort laclosien en vue de peindre des portraits authentiques et non disproportionnés. Comme nous venons de le voir, Riccoboni dédouble ce personnage animé à l'idée d'abuser sexuellement d'Adélaïde, mais aussi prêt à abandonner ses rêves de grandeur au profit de l'amour véritable. C'est une attitude que Laclos escamote vis-à-vis de Cécile de Volanges. La distinction est claire: Danceny lui apporte la déférence de l'amour sincère alors que Valmont ne se complaît que dans l'observation de sa chute. Par conséquent la dimension 
duelle de Cressy le rend moins efficace qu'un vrai Valmont dans l'action du vice et ne le rend pas suffisamment galant pour lui attribuer l'héroïsme affecté de Danceny.

\section{De Raisel à Tourvel}

14 Mais étrangement, Laclos ne craint plus la nécessité d'une métamorphose dans le choix de ses victimes et respecte celles dépeintes par Riccoboni. Le portrait de la comtesse de Raisel reste de loin le plus saisissant dans la ressemblance. Passons sur la proximité frappante qui existe entre leurs patronymes, pour établir la métamorphose déroutante de Raisel en Tourvel. Mme Riccoboni décrit Raisel ainsi :

La comtesse, veuve depuis deux ans d'un mari qu'elle n'aimait pas, [...] entrait dans sa vingt-sixième année ; sa taille était haute, majestueuse ; ses yeux pleins d'esprit et de feu; une physionomie ouverte annonçait la noblesse et la candeur de son âme ; la bonté, la douceur et la générosité, formaient le fond de son caractère ; incapable de feindre, elle l'était aussi de concevoir la plus légère défiance. ${ }^{40}$

Si la présidente de Tourvel n'est pas veuve, l'absence de son mari semble toutefois être un poids pressenti par Valmont : «Quelle étonnante sensibilité ne faut-il pas avoir pour [...] aimer un être toujours absent? $»^{41}$. Le lecteur apprend son âge à la lettre V, Mme de Tourvel a vingt-deux ans. Valmont peint le portrait d'une femme naturelle, «toute parure lui nuit; tout ce qui la cache la dépare $»^{42}$. Mme de Tourvel «est vraiment ravissante » et (le vicomte poursuit sa description physionomique) «n'a point, comme nos femmes coquettes, ce regard menteur qui séduit quelquefois et nous trompe toujours ». À l'instar de Riccoboni qui donne à son héroïne une « âme » candide et bonne, on retrouve chez la présidente "l'image d'une gaieté naïve et franche ! » Il s'attarde aussi à expliquer comment "auprès d'un malheureux qu'elle s'empresse de secourir, son regard annonce la joie pure et la bonté compatissante $»^{43}$. Toutefois, ces portraits idylliques de femmes vertueuses, cachent une capacité «de concevoir la plus légère défiance ».

Mais cette carapace une fois percée nous révèle deux femmes qui savent se sacrifier par amour. Riccoboni dévoile le dévouement de la comtesse lors de l'aveu : «Oui, c'est moi, lui dit-elle, qui ai désiré votre amour [...]. Qu'il m'est cher, cet amour! $»^{44}$. Une dévotion absolue que l'on retrouve chez Tourvel qui aime passionnément Valmont, ce qui la pousse à dire à son amie : «j'aime, oui, j'aime éperdument $»^{45}$. La passion des héroïnes montre un amour parfaitement consacré à l'être unique, un amour dominé par l'oubli de soi. Cette attitude frappante chez Raisel vient créer un décalage significatif dans son couple puisque Cressy sait parfaitement qu'en l'épousant il choisit «l'amour et la fortune $»^{46}$. Lorsque Mme de Tourvel séduit Valmont par la sincérité de son amour, Mme de Rosemonde lui rappelle que «l'homme jouit du bonheur qu'il ressent, et la femme de celui qu'elle procure $»^{47}$. La Présidente écrit d'ailleurs à Mme de Rosemonde : «C'est donc à votre neveu que je me suis consacrée; c'est pour lui que je me suis perdue. Il est devenu le centre unique de mes pensées, de mes sentiments, de mes actions $»^{48}$. Toutefois, leur trop grand amour pour ces deux hommes finit par perdre les héroïnes lorsque les "défiances " sèment le doute dans leurs relations. Un élément perturbateur conforte Mme de Raisel sur les tromperies de son mari. Après avoir entendu les révélations d'Adélaïde au couvent, son attelage manque en chemin de renverser un enfant et, la mère de ce dernier l'invite à se rafraîchir chez elle, lieu où elle découvre la garçonnière de son mari. Au 
début du roman, Mme de Tourvel soupçonne aussi des tromperies et fait suivre Valmont par un espion, cependant la ruse du vicomte rend sa curiosité infructueuse.

Il semble évident aux lecteurs que ces deux personnages féminins auraient pu éviter leurs chutes respectives, puisque de nombreux signes leur avaient indiqué de se méfier. La faute de Mme de Tourvel réside dans la contemplation aveugle vouée à Valmont lorsqu'à trois reprises Mme de Volanges lui intime de rester sur ses gardes : « Sans doute, je reçois M. de Valmont, et il est reçu partout; c'est une inconséquence de plus à ajouter à mille autres qui gouvernent la société $»^{49}$. À défaut d'avoir une amie clairvoyante, Mme de Raisel refuse de voir la réalité qui déchire son couple et la maintient dans une obéissance passive. Elle repousse l'idée que son mari ne l'aime pas et que son mariage repose sur un leurre: «[...] dites-moi, s'écria-t-elle tout en larmes, ce que j'ai fait pour perdre le bonheur de vous plaire $»^{50}$.

Ce serait néanmoins une gageure de voir dans ces deux destins féminins l'incarnation de la morale. Raisel est aussi immorale que Tourvel, dans le sens où, toutes les deux prennent le contre-pied des interdits. Quand Raisel séduit Cressy, elle joue les galantes et s'expose aux préjugés qui cantonnent les femmes au silence. Pis encore, Mme de Tourvel succombe au charme du vicomte de Valmont alors même qu'elle est mariée. L'attitude de ces deux femmes «vertueuses» en apparence rentre dans une dynamique du vice, qui finalement les rend pleinement actrices de leurs chutes. D'ailleurs elles connaissent toutes deux une fin tragique à l'issue des romans. Mme de Raisel se suicide par empoisonnement tandis que Mme de Tourvel sombre dans la déréliction et en meurt. Toutefois la symbolique du trajet que suivent les héroïnes jusqu'à la mort fait l'objet d'une distinction capitale. Le suicide donne au personnage de Raisel un ultime contrôle sur sa destinée; la mort de Tourvel en pénitente accablée relève au contraire d'une démarche plus cynique. Elle meurt exactement comme ces femmes que Merteuil avait accusées de confondre « sans cesse l'amour et l'Amant ; qui dans leur folle illusion [...] ont pour le Prêtre, le respect et la foi qui n'est dû qu'à la Divinité $»^{51}$.

\section{Le sort des ingénues}

Selon nous, Laclos trace les contours de Cécile d'après le patron établi par Riccoboni avec le personnage d'Adélaïde du Bugei. La structure générale de leurs physionomies est aussi saisissante dans leurs symétries que celle de Mmes de Raisel et de Tourvel. Lors de la description de son héroïne, Riccoboni écrit : «Adélaïde du Bugei n’avait guère que seize ans ; [à] tout ce que la jeunesse peut donner de fraîcheur et d'agrément [...] elle joignait ce charme inexprimable que donnent l'innocence et l'ingénuité [...]. M. du Bugei venait de la retirer de l'abbaye de Chelles, dans le dessin de la marier " ${ }^{52}$. L'incipit des Liaisons s'ouvre sur une histoire analogue. Promise en mariage au comte de Gercourt, la jeune Cécile, âgée de quinze ans, vient juste de sortir du couvent. Laclos s'empare du personnage d'Adélaïde et le métamorphose encore une fois dans un contexte libertin, mais l'issue tragique de leurs destins respectifs sera la même. Riccoboni choisit un lieu clandestin, « un feuillage épais " ${ }^{53}$, pour faire subir à Mlle du Bugei une tentative de viol de la part de Cressy.

Retranscrit dans un décor plus intime, cet épisode célèbre est transposé dans Les Liaisons dangereuses. De peur de susciter les soupçons, lors de la remise des lettres interdites de Danceny à Cécile, Valmont demande à la jeune femme la clef de sa chambre pour en faire un double, afin de rendre l'échange des lettres moins flagrant. Si le drame reste le même, Laclos y ajoute les railleries du vicomte : «Sans doute on ne lui a pas bien appris dans son 
couvent, à combien de périls divers est exposée la timide innocence, [...] portant toute son attention, toutes ses forces, à se défendre d'un baiser, qui n'était qu'une fausse attaque, tout le reste était laissé sans défense... ${ }^{54}$. L'ignorance de Cécile devant son agression la différencie d'Adélaïde qui, bien que naïve, résiste à l'assaut. Cette agression est la suite logique de leurs comportements. Les deux jeunes filles s'affranchissent de l'autorité parentale, M. du Bugei et Mme de Volanges destinent leurs filles à un mariage arrangé. Les conséquences de leurs désobéissances seront identiques. Adélaïde, mêlée à un jeu de lettres qui crée un quiproquo, « entra au noviciat $»^{55}$; alors que Cécile s'enfuit à la fin du roman pour devenir religieuse. Sa mère avertie par le couvent se confie à Mme de Rosemonde : «je ne verrais pas sans peine, et même sans crainte, ma fille embrasser cet état $»^{56}$.

21 L'effarement de Mme de Volanges est identique à celle de Mme de Raisel et de M. du Bugei lorsqu'ils apprennent la prise de voile d'Adélaïde : «Mme de Cressy s'affligea du parti que prenait Adélaïde ; elle craignit que ses sentiments pour le marquis ne l'y eussent déterminée $\aleph^{57}$. Certes, le thème du couvent, comme l'affirme Jean Leduc, est un thème récurrent dans le roman du XVIII ${ }^{e}$ siècle : la grille, les macérations et le bien-fondé des vocations des héroïnes sont à l'époque un outil narratologique permettant d'arracher les jeunes filles des griffes du mariage forcé ${ }^{58}$. Toutefois, la concomitance des événements employée par Laclos dans son récit, le rapproche fortement de l'histoire de Mme Riccoboni.

Un synchronisme est reproduit jusque dans les plus minces détails de son roman. Notons brièvement l'importance des témoins abusés et des personnages secondaires reproduite dans les deux récits. Une seule fois mentionné dans l'Histoire de M. le marquis de Cressy, le comte de Saint-Agne « auquel [Adélaïde] était destinée » originellement, annonce le rôle de Gercourt, prétendant officiel de Cécile de Volanges. De même que, respectant un schéma propre à Riccoboni, Laclos va développer la complicité des domestiques dans son intrigue. Le personnage d'Hélène par exemple, femme de chambre d'Adélaïde, devient une complice qui envoie les billets de sa maîtresse au marquis. Décrite comme une « basse créature ${ }^{59}$, Hélène se montre tout aussi dévouée à Cressy qu'à Adélaïde. Ce même personnage ressurgit dans Les Liaisons dangereuses avec Julie, la femme de chambre de Mme de Tourvel, aisément corrompue par le chasseur de Valmont. Azolan devient son amant afin que Valmont obtienne des informations sur la Présidente. Au total, cette galerie de portraits compose une série d'identités gémellaires qui ont définitivement pu constituer autant d'ébauches de plans, d'histoires, de trames et d'intrigues pour Laclos.

\section{Conclusion}

$\mathrm{Au}$ fil de ce dialogue sur les influences de Mme Riccoboni, soulignons que l'étude de Migeot parachève en quelque sorte la nôtre. Dans son analyse de la correspondance entre Riccoboni et Laclos, il relève toutes les "stratégies textuelles " ${ }^{60}$ qui dépassent le romancier dans les réponses qu'il formule à sa contradictrice ; de telle façon qu'un jeu de miroir imitatif émerge dans ces lettres de 1782, notamment par les nombreuses reprises qu'il fait des expressions de l'auteure ou encore par le recours aux italiques venant refléter ses propos. Cependant la présente enquête apporte un complément à la question que Migeot laissait en suspens :

Une première question, que je me pose et que je laisserai ouverte, serait de savoir si

le zèle argumentatif que déploie Laclos sur ce point, son désir d'occuper tout le 
terrain et de ne laisser aucun argument sans réponse [...] ne serait pas un signe de sa faiblesse sur ce point, une forme de dénégation étayée par cette défense véhémente. ${ }^{61}$

À l'aune de la symétrie existant entre les deux fictions, nous comprenons mieux que ce "zèle argumentatif» cacherait le danger de se faire suspecter au sein d'une correspondance dont il perd rapidement le contrôle lorsque les questions qui lui sont posées deviennent de plus en plus subversives ${ }^{62}$. Ajoutons que Riccoboni insiste sur la notoriété de Laclos en répétant «que tout Paris s'empresse à [le] lire, tout Paris s'entretient de [lui] " ${ }^{63}$. D'où peut-être l'envie de défendre son ouvrage aussi ardemment de peur de périr comme la marquise, que Riccoboni mentionne d'ailleurs cruellement en achevant cet échange, puisqu'elle finit par le pardonner sans pour autant le laisser espérer qu'elle se fasse " arracher les yeux $»^{64}$ pour lui. Toutefois, il serait faux de dire que Laclos craignait de passer pour un plagiaire, sa peur résidait ailleurs: sans doute se plaçait-elle dans la gêne d'avoir été percé à jour par l'un de ses maîtres, jubilant devant cet embarras.

Pour cette fidèle lectrice de Hume, la découverte des Liaisons dangereuses a certainement suscité une association d'idée avec son roman de 1758. Si Laclos évoque fréquemment $L a$ Nouvelle Héloïse et Clarisse comme lectures favorites de ses personnages, l'influence de l' Histoire de M. le Marquis de Cressy n'est sans doute plus à négliger dans la liste des sources fondatrices de leurs personnalités. Selon nous, la querelle cache le réel désir de reconnaissance de Mme Riccoboni qui, sans se sentir nécessairement lésée, a pu implicitement satisfaire une envie plus personnelle de régler ses comptes avec un homme qu'elle considère comme un petit-fil ${ }^{65}$. Pour mettre un terme à ce jeu de lettres, Laclos joue sur cette corde sensible et désamorce subtilement le brûlot lancé par la romancière en lui exprimant son admiration. En fin de compte, la querelle des Liaisons ne débouchera pas sur une dispute plus conséquente entre les deux auteurs, puisque seulement huit lettres composent cette correspondance. De guerre lasse et campant sur ses positions Mme Riccoboni préfère conclure ce débat par l'envoi d'un ultime message à Laclos : «Ainsi, Monsieur, après un volume de lettres, nous nous retrouverions toujours au point d'où nous sommes partis " $"$.

\section{NOTES}

1. Entre autres, voir les articles de Champfleury, "Correspondance inédite de Laclos et de Mme Riccoboni », Revue de Paris, 25 sept. 1864, p. 572-585 ; J. Vanpée, «Dangerous Liaisons 2 : The Riccoboni-Laclos Sequel », Eighteenth-Century Fiction, no 9, octobre 1996, p. 33-44 ; M. CharrierVozel, «Féminin et masculin: la pluralité des genres selon Mme Riccoboni et Choderlos de Laclos ", dans Féminité et masculinité dans le texte narratif avant 1800. La Question du " gender ", S. van Dijk et M. van Strien-Chardonneau éd., Louvain, Peeters, 2002, p. 245-246.

2. Pierre-Ambroise-François Choderlos de Laclos, Les Liaisons dangereuses, Paris, Gallimard, «Bibliothèque de la Pléiade », 2011, p. 463. Dorénavant nous ferons référence à l'œuvre et à la correspondance par l'abréviation Liaisons.

Revue italienne d'études françaises, 5 | 2015 
3. «Cette femme écrit comme un ange, c'est un naturel, une pureté, une sensibilité, une élégance, qu'on ne saurait trop admirer », D. Diderot, Euvres Complètes, Paris, Garnier, 1875-1877, t. VIII, p. 465.

4. Laclos, Liaisons, cit., p. 468.

5. Ibid., p. 466. C'est moi qui souligne.

6. E. A. Crosby, Une romancière oubliée, Madame Riccoboni, Genève, Slatkine Print, 1970, p. 141.

7. P. de Marivaux, La Vie de Marianne, préface de J. Janin, Paris, Charpentier éditeur, 1842, p. 14.

8. Nom de jeune fille de Marie-Jeanne Riccoboni.

9. G. May, Le Dilemme du roman au XVIII e siècle, 1715-1761, Paris, PUF, 1963, p. 254.

10. M. Reid, "Note", dans Marie-Jeanne Riccoboni, Histoire de M. le marquis de Cressy, Paris, Gallimard, «Folio », 2009, p. 11-12. C'est moi qui souligne. Dorénavant nous ferons référence à cette œuvre par l'abréviation Cressy.

11. P. Fauchery, Destinée féminine dans le roman européen du XVIII ${ }^{e}$ siècle, 1713-1807. Essai de gynécomythie romanesque, Paris, Armand Colin, 1972, p. 11.

12. Riccoboni, Cressy, cit., p. 62.

13. Ibid., p. 64 .

14. Ibid., p. 42.

15. Ibid., p. 70.

16. Laclos, Liaisons, cit., p. 466.

17. Ibid., p. 468.

18. C.-J. Dorat, Les Malheurs de l'inconstance (1772), dans Romans libertins du XVIII e siècle, cit., p. 900.

19. Voir aussi l'étude de R. Mauzi, "Sur la genèse des Liaisons dangereuses", Lettres et réalites. Mélanges de littérature générale et de critique romanesque offerts au professeur Henri Coulet par ses amis, Aix-en-Provence, Université de Provence, 1988, p. 191-226.

20. Laclos, Liaisons, cit., p. 463.

21. Ibid., p. 469.

22. Voir sur les origines de Mme de Merteuil les études de L. Versini, Laclos et la tradition. Essai sur les sources et la technique des Liaisons Dangereuses, Paris, Broché, 1968 et de C. Jacot Grapa, Dossier dans Les Liaisons dangereuses de Choderlos de Laclos, Paris, Gallimard, « Foliothèque ", 1997.

23. Laclos, Liaisons, cit., p. 466.

24. Ibid., p. 469.

25. Ibid., p. 463.

26. Ibid., p. 465.

27. Riccoboni, Cressy, cit., p. 28.

28. Laclos, Liaisons, cit., p. 205.

29. Ibid., p. 204.

30. Ibid., p. 210.

31. Riccoboni, Cressy, cit., p. 79-80.

32. Laclos, Liaisons, cit., p. 469.

33. N. Elias, La Société de cour, Paris, Champs-Flammarion, 1985, p. 99.

34. Laclos, Liaisons, cit., p. 465.

35. Molière, Dom Juan, Acte I, scène 1, v. 77.

36. Riccoboni, Cressy, cit., p. 21.

37. Ibid., p. 22.

38. Laclos, Liaisons, cit., p. 140.

39. Riccoboni, Cressy, cit., p. 31.

40. Riccoboni, Cressy, cit., p. 23.

41. Laclos, Liaisons, cit., p. 27.

42. Ibid., p. 26.

43. Ibid., p. 26-27, pour cette suite de citations. 
44. Riccoboni, Cressy, cit., p. 71.

45. Ibid., p. 28.

46. Ibid., p. 62.

47. Laclos, Liaisons, cit., p. 362 .

48. Ibid., p. 357.

49. Ibid., p. 80 .

50. Riccoboni, Cressy, cit., p. 92.

51. Laclos, Liaisons, cit., p. 204.

52. Riccoboni, Cressy, cit., p. 23-24.

53. Ibid., p. 57.

54. Laclos, Liaisons, cit., p. 254.

55. Riccoboni, Cressy, cit., p. 76-77.

56. Laclos, Liaisons, cit., p. 448.

57. Riccoboni, Cressy, cit., p. 77.

58. J. Leduc, "Le clergé dans le roman érotique du XVIII" siècle ", dans Roman et Lumières au XVIII siècle, Centre d'études et de recherches marxistes, Paris, Études sociales, 1970, p. 341-349.

59. Riccoboni, Cressy, cit., p. 47.

60. F. Migeot, « Rapport de places et imaginaire dans les lettres de Laclos et Mme Riccoboni », Semen [En ligne], 20 | 2005, consulté le 2 décembre 2015, URL : http://semen.revues.org/2038 § 37. 61. Ibid., § 36 .

62. Dès la lettre III, Mme Riccoboni entre dans le jeu de Laclos et le pousse dans ses retranchements: "Vous avez la fantaisie de me persuader, même de me convaincre par vos raisonnements, qu'un livre, où brille votre esprit, est le résultat de vos remarques et non l'ouvrage de votre imagination. N'est-ce pas là votre idée ? ». Laclos, Liaisons, cit., p. 471.

63. Ibid., p. 466.

64. Ibid., p. 473.

65. Dans la cinquième lettre Riccoboni fait état de son âge avancé et rappelle non sans humour à Laclos qu'elle est « une femme dont il a pu entendre parler à sa grand-mère ! ». Laclos, Liaisons, cit., p. 471.

66. Ibid., p. 479.

\section{RÉSUMÉS}

Que nous reste-t-il à apprendre de la correspondance entre Mme Riccoboni et Choderlos de Laclos? Commencé par la romancière après la parution des Liaisons dangereuses en 1782, cet échange de lettres prend vite l'aspect d'une querelle sur le roman en général. À trop vouloir isoler le conflit manifeste qui anime les épistoliers autour de la sulfureuse Merteuil, on néglige peut-être que la subversion du roman libertin puise toute sa force dans les règles du roman moral. Dès lors, le roman de Laclos - forme dégradée ou dérivée du genre - se fait souvent l'écho de celui de Riccoboni en ceci qu'il en bouleverse le fond et en préserve les formes. Cette étude repose sur une lecture croisée entre l'Histoire de M. le marquis de Cressy écrit par Mme Riccoboni en 1758 et Les Liaisons dangereuses de Laclos. Nous nous proposons d'aborder sous un autre angle les enjeux de la pomme de discorde entre les deux auteurs et d'apporter une analyse sur la logique 
qui gouverne la construction des personnages. De la comtesse de Raisel à Madame de Tourvel : quelles cohérences président jusqu'à la mort?

INDEX

Mots-clés : Riccoboni (Marie-Jeanne), Laclos (Pierre-Ambroise-François Choderlos de), correspondance, intertextualité, roman moral, roman libertin 